\title{
FRECUENCIA DE LAS PARASITOSIS INTESTINALES EN LA POBLACION INFANTIL DE QUITO
}

\author{
Dor. MAGDALENA RHEA C. y Dr, JOSE CASTRO C.
}

id parasj tismo in lantil tiene una am. plis gialidad en en tordis el mundos con

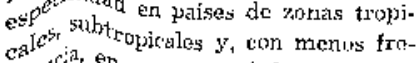

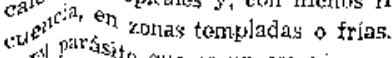

f) parsitits que es un ser viru, aninit ${ }^{D}$ regetal, pasa una parte o la totald intes sil existeneid en la superfin ce diter do ofro ser vivo, bitelógi-

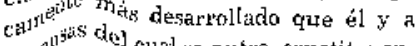

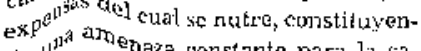
do $u^{13}$ to bueraza constante para lie sa-

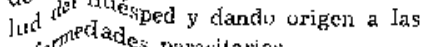

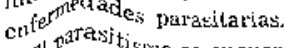

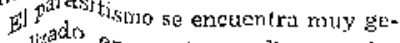
netitition en ruestro medio y varios facto do recorocen como determinatite te trato hecho. La finalidad del preti colyajo sue la de reilizar un e.5 t. di a citos parativo de la incidencia

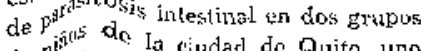
de river Ia ciudad do Quito, uno peribe a la tye porplar.$$
\text { 1) }
$$$$
\text { Ei fipo represcntativo de la clase }
$$

media estuvo constituído por 250 niños asisientes al Jardin de Infantes "Mexcedes Nobua", el lanto que el segundo grupu estuvo formado por 256 niños provenientes de hogares de escasiss recursos económicos $y$ asistentes a Ja Casa Maternal "Carolina T'erán", adserito a la Direccion General de Hogares de Frotección Sircial.

De coda uno de estos rix̌oz fue tumada una muestra de heces, en lá cual se realizé el examen coproparasitario respectivo.

\section{RESULTADOS}

Herros encontrado zina fenctencia clevada de núos parasitados: monoparesitadus, biparasitadiss, iri y ietra pum rasitútos. Ln mayor parte de estus ni$\overline{\mathbf{n}} \mathbf{3}$ presentan un estado de destulutición total, contrastando con un esenso porciento de niños no parasitudos quo claramerte demuestrat su bucn estado física te intelectua]. Los resultados abtenidos be resumen en las Tablas I y II. 
TABLA I

INCIDENCIA DE PARASTTOSIS INTESTINAI, EN NIŇOS WE LG: JARDINES "MERCEDES NOBOA" Y "CAROIANA TERAN" JE QLITO, 1963

\begin{tabular}{|c|c|c|c|c|}
\hline & \multicolumn{2}{|c|}{$\begin{array}{c}\text { Niños de clase } \\
\text { media }\end{array}$} & \multicolumn{2}{|c|}{$\begin{array}{c}\text { Siños de class: } \\
\text { popular }\end{array}$} \\
\hline & $N^{\prime !}$ & $1 \%$ & $\mathbb{N} !$ & 拝 \\
\hline NITGATIVOS $\ldots \ldots \ldots$ & 77 & 30,8 & 48 & $\{8,75$ \\
\hline $\begin{array}{l}\text { EOSITIVOS } \\
\text { Munoparasitados .... } \\
\text { Puliparasitados .... }\end{array}$ & $\begin{array}{l}78 \\
95\end{array}$ & $\begin{array}{l}31,0 \\
38,2\end{array}$ & $\begin{array}{r}76 \\
132\end{array}$ & $\begin{array}{l}29,65 \\
51,6\end{array}$ \\
\hline T OTAL............. & 250 & 100,0 & 259 & 100,0 \\
\hline
\end{tabular}

Wil grado mayor de infestación paraiilaria herugs eucontrado en la Casä ivaternd "Carolinat Tétán"; indudaHbenente allí cruncurren niños de ruunsos socio-cenómices deficientes $y$ Iuाy malos.

La infestación de A. histolítica en e] "Curolina Terán" coxiesponde a un litr: y en e] "Mercedes Noboa", a un int $4 \%$.

Do igual manora la infestación con «kus parásitos si bien no es elfovada, peco si de consideración.

\section{Fuclores detcrniutantes de la clevada} incidoncia de parasitosis intestjoal en muestro medio.-Según pstakísticas $y$ talos tomiados de estudios realizados cll muestro país, un buen porciento de la mortalidad infantil sic debe en enfermetlades infecciosas $y$ parasitarias. î. lislad infantil muy elevado, indice que ma: da la medidit de Ias condiciones sunitarias y sociomeconomínus de nuestra rogion. Naturalncate, esias condiciunes son siempre mejores en las árens: urbanas on relatión coul lis zonas rurales, pues en ćstis últimas hay un brjo niwel de coluessión sanjitaria $y$ eultural.

En la ciudedd de Quito, podemess analizar las tondiciones sanitaries teniand, el cuenta 3 sectores: sur, cetstral o ccmercial y hoste o residencial. La distribución demográfítia on estos 3 rentores es marcadamente desigual con respecto al perímels'o o zomar que setepan, Asi, el scetor stu, densamente pcblado por miles de familias de recursos: eeonómicos reducidos, veupa una área urbane relativamentや pequeñ:i.

Los problemas mus comures a sus habitantos son: estrecher de la vivierida, ralta de servicios higiérilcos, falta de canalizaciones y filtá de ugua putable, pucs hay escasez de la misma en estos sectores superpoblados. 
TA[3]A :I

PARASITOS IDENTIFICADOS EN LOS EXAMFNES COPROPAKASITARIOS PERTENLCIENTKS A NIFOS DE LOS JAMUINES "MLRCEDES NOBOA" Y "CAROLINA 'IERAN" DE, RUITO, 1963"

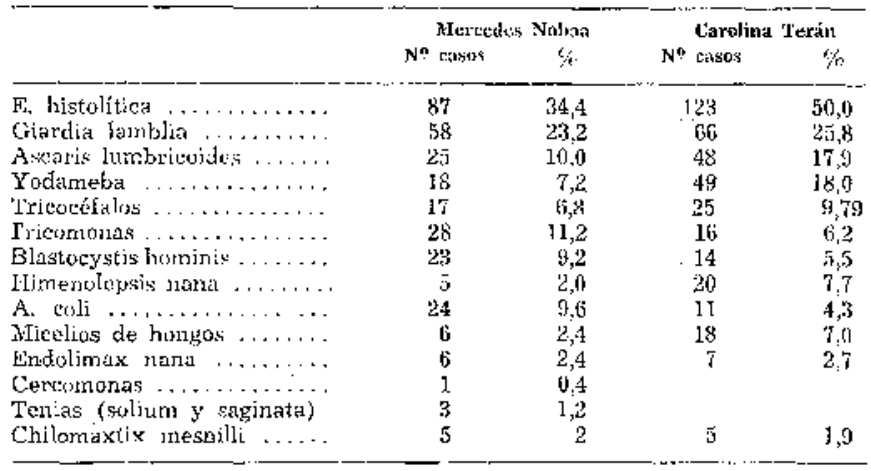

El sector central está ocupado principalmente por el comercio de la ciudadi al problema higiénico en cste: sector tadica cn la presencia de los vendedores ambulantes. El1 estos ú]timos anos se wherva un l'onsiderable aumento de jersonas que se dedican al comercin do aljmentos en silios públicas y calles de la ciudad, contrariando las disposieiones del Códjgo Sanitario de expendio de álimentos. Aproxinadamente en Quito existen tres mil personas que realjzan este comercis; sun gentes de escusa cultura, (gut: constifuyon un vexdadero poligro para la salud pública, afcetando aúr el ommato $y$ dignichad de la cinudated.

El sectur norte o residencial es cl más amplio; el problema de la viviendis no está strilucionado completamente, vero no presenta las concliciones det:cientes de] sector sur. La miyis parte de las familias viven un casas o apdrtementos eun bucua ilumination, vezn 1:lactión y condicionss higiénicas $y^{\prime}$ saנitarias bиелаs.

Un aspecto fundamental en cuarslu se: refiere a la tondición sanitavia de la ciudad, es la recolección de basura. Fin nuestro medid, d oriçen y purceinento de basuras recolectadas son Ios sigutientes: transcúntes $10 \%$, verdodores imbulantes 20\% y los que arroj vías puiblicas y que no fueron entragadas a lis carros recolectores, $70 \%$.

La cuntidad diaria de las basurak pe- 
lisrußas quc se rengen en las calles ascicnden a 600 quintalesi, cantidad su. utamente elevada un comparación con las que produced thas ciudades, En utudades alemanas el promediry amual jor persoua es de $1 / 2$ kilo y en Quito, es de 25 kilos: o sca 50 yecess más.

\section{CONCIUSAIONES}

1) Wl medio fidrorable parit el desaro!lo de los parásitos trate como cunsecuencia que uusitra población pre. escoliur y escrolar suluil constanterrence de cortínuas enfermedades parasititrias, que fata]monte suan acerituando cala vez mús, debjdo a la falta de tonocimiento de lá nigitule! personâ].

La higjene pertonal, lit higicne ambinntia y la higiene de la conunúdad, se oumplementan y se ayudarı part levantar el sivel cuitural de un pueblo.

2) Las instituciones $y$ autoridades sanitarjas, deberán cumplir una camjaña muy intertsa y contínita pitra haeer conciencia en la clase medirs y popular, ds la importaucia que tiene $\mathrm{cr}$ la salud del individuo: la higiene persuna], la manera de prepatur los aljmentos, la manera de conservarlos en ol medio familiar y la manera de scrvirlos.

3) La falta de medios económicos en nuestra chase popular, hace que vivan t.u uma sifuación lamentable, sin Ias condicancs sanitarias recesurias y que por sus propios medios no pueden, ni putfrán superarte, sino e de Ias instituciones futuermamentales: que deben hares obeal positiva sn el verdadero pueblo mejoraulos su viviers. da yr su 22jure! enlituroI.

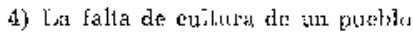
hace que mire eon poca presucupación las consevupucins fatales que l lewitn crum las enfermedades pa:asitarias; : 1 lamente es necesario que en el medio [am:liak se concuentra nue persuna paras:Ladí, paria jie por la falla de pretcrici. vión y las condiudenes sinjarias nuty deplorables, después de poco tienpo sear portadores de parásitos, sino toda la familia: por lo mencos un buers yorconlinje de ella.

5) Del Ltabrajo realizado prodencos deducir, quo el niz̄n se encגuntra más expuestu a las infecciuses parisitarias desde la codad de dós añus y medio is einco años y medio, eit decir deste la epoca en que trata de independizarso de his cuidadis rle otra porsona hasia los comienzos the la edad escular, en que va exdquiriendo una vikla sistertatizada.

\section{RESUMFN}

Se realizó un estudio eomparälivo d:! la incidencia de patasitosis jnicstinat? en dos grupos dn nin̄os, de diferentu? condición cconónica; uno sle la clase medir $y$ otro de lat clese populitr.

Ln los diñ̂́os de Is clase med:a se encontró $30,8 \%$ fie cases negatives $;$ $69,2 \%$ de positivos, lrevte a 18,75\%i. de resultados therativos y $81,2,5 \%$ de posjtivos en los niños de la clase popular

Los parásitus más frectuontes, en or- 
den de importancia, fueron los siguientes: F, histolítica, giandia lamblia, áscaris sumbrirosides, tricocéfalos y tricumumas.

La mayor ancikencia te parasitiusi; en los niños de lo clase popular se debe probablemente a las deficient es condiciones de salubridad en que cllos st descnvuelven. Fil puliparasitismo, a su ver, inluye nocivamente no sólo sobre el desarrollo físieco, sino lambién sobre la rapac:dad intlectual de estos ת:s̆os.

\section{SUMMARY}

A comparative study on the incidence of indestinal parasitosis was made in two groups of ehildren of different ceoucmical conditions; one group re- presented the midctle class and the wther, the lower class.

In the middle rlass grtup, $30.8 \%$ of sergative cases and $69.2 \%$ positive $6 a-$ ses werc found, while in the lower class group, 18.75\% were nogative atud $81.25 \%$ were positive.

The most frequenlly found parasites, listed acording to their importance, were: E. histulitica, Giardia Iamblia, Ascaris lumbrieoides, Trichocephalus, Trichomonas.

The greater incidence of parasilusis in children of the low economical grouj is probably due tor the cleficient sanitaky eonditions in which they develop. The poliparasilism found in this group of children influcoces harmfully not only their physical devalopemert, but. also jn their intelectual capacily.

\section{LI.CHRACIONES INTESTINALES POK DIURETICOS CON POTASIO}

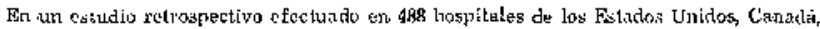
Sudamćrica, Etıupa, Afries, Aust ralia y Nubva Zelandia, se ha encontrado una ciusuístico de 484 pacientes con tuceraciones en el intestino delgith, de los cuales el 57 o hahin

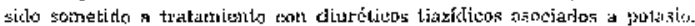

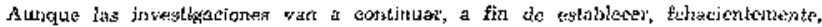
si la asociación de diurético tiknídico con protasio puede ser realmerit vatsia de ulceración

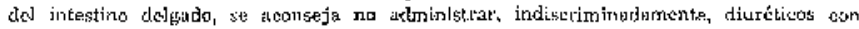

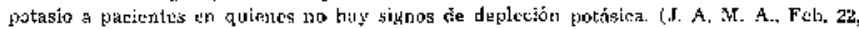
1965). 